\title{
Health Promotion Sciences
}

National Cancer Institute

\section{Source}

National Cancer Institute. Health Promotion Sciences. NCI Thesaurus. Code C18833.

Research into how best to encourage individuals to take control of their health and to engage in healthy practices. 